\title{
Pictographs, Ideograms, and Emojis (PIE): A Framework for Empirical Research Using Non-verbal Cues
}

\begin{abstract}
We propose an empirical framework to understand the impact of non-verbal cues across various research contexts. A large percentage of communication on the Internet uses text-driven non-verbal communication cues often referred to as emojis. Our framework proposes two types of factors to understand the impact of emojis. The first type consists of pictographs, ideograms, and emojis (PIE) factors such as usage, valence, position, and skin tone, and the second type consists of contextual factors depending on the research context, such as fake news, which has high social impact. We discuss how the effect of PIE factors and contextual factors can be used to measure belief, trust, reputation, and intentions across these contexts.
\end{abstract}

\section{Introduction}

Communication is fundamental to human existence. Technology has changed rapidly over the last two centuries and forced researchers to revisit the fundamentals of communication and understand this concept in greater detail time and again. From radio to Internet, new aspects of communication have brought new opportunities and challenges for research. Although managing propaganda using advanced communication channels since World War II has been a key focus of the social sciences [1], the rise of the Internet and the associated increase in the use of social media platforms has brought many challenges for the IS community, including fake news, cyberbullying [2], online shopping [3], and information security.

There are two key forms of communication- verbal and non-verbal [4]. Emojis, also referred as ideograms or pictographs, are visual symbols that define an idea but are not tied to any specific language, and are a part of non-verbal communication. Emoji is a Japanese word meaning picture-word (e-moji) [5]. Emojis were added to the Unicode system in 2009 by the Unicode consortium [6]. Nearly $90 \%$ of the characters on the internet are Unicode characters [6]. The rising popularity of emojis can also be estimated by the historic moment of 2015, when the pictograph was declared the word of the year by Oxford Dictionaries ${ }^{1}$. This was the first time a pictograph was chosen over a textual word, indicating the rise of ideogrammatic icon usage on the Internet. Additionally, in 2016 alone, an estimated 2.3 trillion mobile messages used emojis ${ }^{2}$.

Emojis encourage positive behavior, such as increased purchase intention online [7], but are also used for negative purposes, such as online advertising by human traffickers [8]. Moreover, under different contexts, emojis display different emotions [9]. This varied usage of emojis across different domains raises several questions about their impact and purpose. Given their popularity and diverse purposes, it is important to study the usage of this form of communication in greater detail. We propose a framework to study the impact of emojis across several domains using high level factors labelled as PIE factors, which are characteristics of emojis (e.g., emoji valence, emoji position), and contextual factors that apply to the domain of interest (e.g., type of product- utilitarian versus hedonistic, type of information- true versus fake). Although there are several reasons for using emojis in a communication phrase, this study is concerned with the consequences of such usage. For example, emoji usage may moderate effectiveness, reach, or perception of the sentiment of the message. The remainder of the paper is divided into 4 sections. First, we revisit the previous literature and background; second, we discuss PIE factors and the overall framework; third, we discuss three research contexts and possible predictors in those contexts; fourth we discuss the framework's implementation in the fake news context in detail; and last, we conclude the paper with overall takeaways.

\section{Theoretical Background}

\subsection{Elements of communication}

Verbal communication consists of face to face conversations where the expressions of the participants of the communication are visible to one another [10]. These expressions indicate emotions and are capable of altering beliefs and intentions [7, 10].

${ }^{1}$ https://languages.oup.com/word-of-the-year/2015/

2 https://cdn.emogi.com/docs/reports/2016_emoji_report.pdf 
The other form of communication, which is very popular on the Internet, is non-verbal communication. Non-verbal communication takes place mostly in the form of text on the Internet. Emails, text messages, online posts, and tweets are some examples of nonverbal communication $[11,12]$. On the Internet, nonverbal communication is divided into two key areas -computer mediated communication or CMC (e.g., email, messages) and social networking sites (SNS) communication (e.g., tweets, Facebook posts) [13]. Although CMC has existed for a while, SNS is a relatively new phenomenon [14]. This distinction is important because CMC typically involves fewer parties, as it is a direct communication, while SNS involves many more parties, and it is therefore more important to understand the tone/emotional load of the communication differently in each context.

A key difference between verbal and non-verbal communication is that non-verbal communication is not capable of effectively expressing emotions [14]. Additionally, insufficient non-verbal cues reduce CMC's capability to form interpersonal relationships $[14,15]$. However, intelligent usage of keyboard characters from the early days of computer-based communication gave rise to paralanguages [16]. It started with the simple arrangement of letters (e.g. using capital letters to loudly express a word) and emoticons :-) [17], and later evolved into Unicode characters called emojis $: ;$ [11].

Some key elements of human communication are sender (i.e., the source), receiver (i.e., the destination), and message (i.e., the content). Messages are texts or ordered groups of signs that are meaningful for communicators [1].

Elements of communication theory can be broken down into seven traditions [18]. They have been described as (1) the semiotic- this deals with signs. Ideograms such as emojis fall under this tradition of communication; (2) the phenomenological- this deals with the experiences of individuals; (3) the cyberneticthis deals with the influence of interactive elements on each other; (4) the sociopsychological- this deals with a person's social behavior and other psychological elements; (5) the sociocultural- this is related to how people communicate between themselves rather than focusing on the single individual; (6) the critical- this is related to how power, entitlement, and persecution influence certain forms of communication; and (7) the rhetorical- this is related to making speeches and building arguments communication.

\footnotetext{
${ }^{3}$ https://emojipedia.org
}

\subsection{Semiotics- Pictographs/Ideograms/Emojis}

Semiotics, also referred to as the study of signs and symbols, encompasses a wide range of theories on "language, discourse, and non-verbal actions" [1 p. 45]. Semiotics is broadly divided into three areas [19]. The first area is semantics- related to the meaning of the sign or what it represents in a particular context [20,21]. The second area is syntactics. Here, the relationship between signs is defined [1] as signs are usually not atomic in nature. Compound signs (combination of one or more signs) carry more meaning compared to atomic signs (e.g. a wet floor sign is a combination of a person falling and a straight line representing the floor, and occasionally some small droplets to represent water). The third area of semiotics is pragmatics [22] - related to putting the signs to use into daily life (e.g., traffic symbols like the STOP sign).

Emojis have been divided into eight categories ${ }^{3}$ [6]. A total of 2,382 emojis exist across the eight categories. These categories are (1) Smileys \& People $(1,266$ emojis)- These emojis express different types of faces (e.g., grinning face, sad face, hugging face), people (e.g., man, woman, child, doctor, construction worker), families (e.g., man-woman-girl, man-man-boy, womanwoman-boy-girl), hand gestures (ok-hand, raised hand, Vulcan salute), clothing (e.g. coat, scarf, t-shirt, necktie) and accessories (e.g., ring, lipstick, hat). (2) Animals \& Nature (113 emojis)- These emojis express different types of animals (e.g. see-no-evil monkey, cat, mouse, rabbit) nature (e.g. sun, snowflake, various moon phases, flowers, leaves), and weather (drizzle, snowflake, rainbow) (3) Food \& Drink (102 emojis)These emojis express different fruit (e.g., apple, banana, tangerine), vegetables (e.g., carrot, potato, broccoli), meals (burger, taco, pizza, cooked rice), beverages (e.g., topical drink, beer mug, wine glass), and utensils (e.g., spoon, fork and knife, chopsticks) (4) Activities (60 emojis)- These emojis express different types of sports (e.g. basketball, cricket, person golfing), music, (flute, saxophone, guitar) the arts (e.g. person in suit levitating), hobbies (e.g., thread, yarn, artist palette) and other activities (e.g., circus tent) (5) Travel \& Places (207 emojis)- These emojis express different sceneries (e.g., snowcapped mountain, camping), locations (e.g., national park, Mount fuji), buildings (e.g., hotel, school, factory) and modes of transport (e.g., metro, train, bus ) (6) Objects (162 emojis)- These emojis express different types of household items (e.g., thermometer, radio, alarm clock), celebrations (e.g., gift box), stationery (e.g., books, notebook, scroll) and miscellaneous objects (e.g., abacus, shower, sponge, adhesive bandage) (7) Symbols (205 emojis)- These 
emojis include different types of heart emojis (e.g., blue heart, orange heart, yellow heart), clocks (e.g., clocks with different times), arrows (left, right, end arrow), signs (e.g., zodiac signs, arithmetic signs, atm sign) and shapes (e.g. colored squares, circles) (8) Flags (267 emojis)- List of country flag emojis (e.g. US flag, India flag).

\subsection{Emotion and Emoticons}

Ekman (1992) argues that the fundamental role of emotion is to prepare a person to deal quickly with interpersonal interactions. These interactions stem partially from our biological and personal histories [23]. Ekman (1992) broadly defines six key emotions: anger, fear, sadness, enjoyment, disgust, and surprise. The content (text and emoji) of the tweet has regularly been used by computational linguists $[9,20,24]$ to predict these emotions. There are then five additional emotionscontempt, shame, guilt, embarrassment, and awe which have not been studied in as much detail.

Interactions on the Internet were less emotional in the early days of the Internet [25]. This has been overcome by the use of emoticons [10]. Emoticons are used to express emotions and strengthen the impact of the message being delivered [26]. Emoticons enable exchange of emotions [26] and reduce confusion in the communication [27]. Additionally, in the age of social media, emotions are conveyed through direct and indirect usage of ideograms [28] as well. Several studies have examined this issue from different perspectives. [24] suggest using ideogrammatic icons as function words, while [11] provide an important insight by showing that humans agree more about the sentiment of a message when an emoji is involved. We also see that the effect of emoji is moderated by product type (utilitarian versus hedonistic) on purchase intention, and is stronger for hedonic products compared to utilitarian products. [7]. However, [7] consider the same type of emojis (positive and neutral) for both product types. Emoji usage needs to fit with the advertised product, or else no [7] or negative [29] effects of emojis are possible. We can use new characters with creativity and express emotions in an online social context, which can help us bring it as close as possible to face to face communications [17].

\section{Directions for Empirical Work}

\subsection{PIE Framework and Factors}

Emojis are used in creative ways in conversations. Regular text based CMC reduces impression formation [30] and using nonverbal cues such as emojis aids in impression formation [16]. However, to better understand how emojis can impact attitudes in any study, we must understand different characteristics of emojis. These characteristics, or factors, are central to understanding emojis, how they are used, how they encourage people to share, and how they facilitate communication. In this section, we discuss what we refer to as the four foundational factors of the PIE framework as shown in Table 1. More factors may be considered in future analyses. The overall PIE factors represented as a stack, and the framework are shown in Figures 1 and 2. The PIE stack shows the four PIE factors which can be chosen based on the research question and context. The PIE framework describes how empirical questions can be analyzed and answered to study the impact of emojis under different contexts.

3.1.1 PIE Framework: To utilize the framework effectively, the first step is to choose a context based on researchers' interest. Inspired by the research context, the research question and the phenomenon of interest (dependent variable) must be identified. Examples of phenomena of interest are shown in Table 3. Next, the effective emoji factor must be identified. If the context is studying product consumption across demographics, then the color factor can be chosen to determine the moderating effects of skin tone and identification with the product. Similarly, if the effectiveness of emotions is being studied, then the valence factor can play an important role. After choosing the PIE factor, context factors must be chosen. These factors are predictors or regressors of the phenomenon of interest being measured. Existing literature or theory can also help in choosing contextual factors. Finally, based on the coding and research question, an analysis method must be chosen (e.g., linear regression, anova).

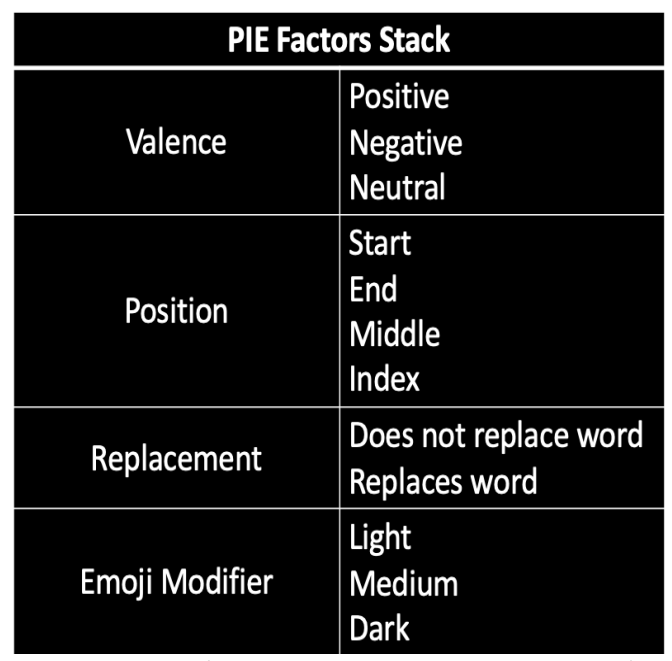

Figure 1. PIE Stack- PIE Factors. One or more can be used in a study. 


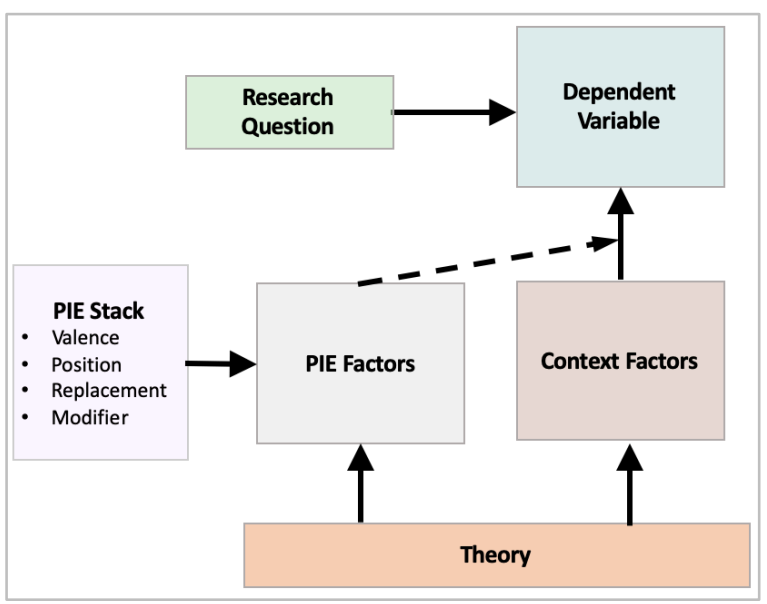

Figure 2. PIE Framework

3.1.2 Valence: This indicates if the emoji is positive, negative, or neutral in nature. Different types of laughing smileys $(;), 8$,$) or non-facial symbols (fo,$

Q28) act as emojis with positive valence, while certain emojis, such as sad or angry faces (궁, -2 ), serve as emojis with negative valence. Several facial and nonfacial emojis that do not express any emotions serve as neutral emojis $(\cdot, \odot, \odot$, It is important to consider the role of emoji valence, as valence is capable of altering the interpretation of the message being communicated [10], especially when the overall valence of the message being communicated is opposite of the emoji valence [31]. Valence can be utilized as a predictor and can be coded into three levels as indicated in Table 1. Emojis with different valence and keywords are shown in Table 2.

3.1.3 Replacement: Each emoji is represented using a keyword that is used to describe the emoji. These keywords are formed by combining a sequence of words. The emoji itself is constructed by combining the Unicode character to describe each item in the sequence. For example, the emoji is a sequence formed by combining Person and graduation cap emoji and is assigned the keyword student. Some research has treated words as speech and the emoji as a gesture, suggesting that exchanging an emoji for a word and vice versa is a universal feature of multimodal communication $[32,33]$. In this example, the emoji can be used in two ways in the message. The first way could be to use the word student and student emoji to create a message (I am a student at the University of Emojiland); the second could be to just use the student emoji without the supporting word (I am a at the University of Emojiland). Both ways are open to interpretation by the readers. While some may find the first usage redundant, other readers may find it helpful, and some readers may have similar experience with the second usage. Existing studies have shown that it takes more time to read text where some words have been replaced by emojis [34]. [34] recommended emojis should enhance but not replace words, indicating the presence of potential cognitive load. In their task, [35] used pictures along with emojis and found their neural network was able to predict the emoji better when the picture was used versus when the picture was not used. This is a complex factor where two levels of replacement exist as shown in Table 1.

3.1.4 Position and Frequency: Emojis can appear at different locations in the text. However, their location and frequency are not random. They appear in welldefined linguistically important positions in the message [36]. They can appear at the beginning of the message ( $\odot$ the game today), or in the middle (I like of with some lemonade), or at the end (Can we watch the match please (30), or at any random position (I think this is business, but who can say (-)) in the message. While the importance of position is recognized in neural networks [9], their use as a neural network feature remains understudied. If researchers want to measure the impact of position and frequency of the emoji in the sentence using linear regression, an absolute index of the emoji based on its position and the number of times it occurs in the message can be used as predictors. If anova or a similar method is chosen for analysis, the predictor can be coded as shown in Table 1.

3.1.5 Emoji Modifier: The Unicode consortium added five skin tones in 2015 inspired by the Fitzpatrick phototype scale [37]. This modifier enables selfrepresentation of a large range of demographics [38]. While other features of emojis discussed earlier have been studied in detail, the effect of skin tone remains an understudied phenomenon. This feature can be used to understand online racism, bias, and hate. While certain emojis like closed fist ( $)$ are associated with darker skin tone, other emojis such as thumbs up ( $\mathrm{d}$ ) are associated more with light skin tones [39] highlighting the significance of demographics and race in emoji usage. Empirical research aiming to use linear regressions can utilize the skin tone scale $(1-5,1$ being lightest and 5 being darkest) as defined by the Unicode consortium [6], a value of 0 can be utilized for no skin tone color. Similarly, if the choice of analysis is anova, skin tones 1-2 can be coded as light, 3 as medium, and $4-5$ can be coded as dark. The effects of this feature can be studied to understand product consumption and learn if certain endorsements using particular skin tones are likely to affect purchase intentions for different product 
types (hedonistic/utilitarian) differently, and to study whether any difference in purchase intention (low/high or continuous) is moderated by the effect of skin tone (either using the linear scale of 1-5, or three levels as described above). In the context of fake news, the impact of skin tone can also be studied when the message is shared online by an endorser.

\begin{tabular}{|l|l|l|}
\hline \multicolumn{1}{|c|}{ Factors } & $\begin{array}{c}\text { \# of } \\
\text { Levels }\end{array}$ & \multicolumn{1}{|c|}{ Level Description } \\
\hline Emoji valence & 3 & $\begin{array}{l}\text { Positive emoji } \\
\text { Negative emoji } \\
\text { Neutral emoji }\end{array}$ \\
\hline $\begin{array}{l}\text { Emoji usage- } \\
\text { treated } \\
\text { consistently } \\
\text { across contexts }\end{array}$ & 3 & $\begin{array}{l}\text { No Change- control } \\
\text { condition } \\
\text { Emoji replaces word } \\
\text { Emoji does not } \\
\text { replace word }\end{array}$ \\
\hline $\begin{array}{l}\text { Emoji position } \\
\text { and frequency }\end{array}$ & 4 & $\begin{array}{l}\text { 0-None, 1-start, 2- } \\
\text { middle, 3-end, 4- Any } \\
\text { index }\end{array}$ \\
\hline $\begin{array}{l}\text { Emoji skin tone } \\
\text { (Depending on } \\
\text { demographics) }\end{array}$ & $3-4$ & $\begin{array}{l}\text { Neutral } \\
\text { (yellow/golden), } \\
\text { White, Black, Brown }\end{array}$ \\
\hline
\end{tabular}

Table 1. PIE Factors: Levels and Description

\begin{tabular}{|l|c|l|}
\hline Valence & Emoji & \multicolumn{1}{|c|}{ Emoji Description } \\
\hline Positive & $\odot$ & $\begin{array}{l}\text { Emojis expressing joy, love, or } \\
\text { tasty food. }\end{array}$ \\
& $\ddots$ & \\
\hline Neutral & $\ddots$ & $\begin{array}{l}\text { Emojis expressing surprise, } \\
\text { doubt, and expressionless face }\end{array}$ \\
\hline Negative & $\odot$ & $\begin{array}{l}\text { Emojis expressing anger, } \\
\text { pouting face, sad cries }\end{array}$ \\
\hline $\begin{array}{l}\text { Non- } \\
\text { facial }\end{array}$ & $\ddots$ & $\begin{array}{l}\text { Emojis expressing rocket, } \\
\text { popcorn- usually used to } \\
\text { denote movies or cinema, fire- } \\
\text { also used to describe something } \\
\text { exciting }\end{array}$ \\
\hline
\end{tabular}

Table 2. Emoji Valence Examples

\begin{tabular}{|c|l|}
\hline$\#$ & Potential Dependent Variables \\
\hline 1 & Emotional arousal and valence \\
\hline 2 & Likelihood of sharing/retweeting \\
\hline 3 & Likelihood of liking \\
\hline 4 & Trust in information \\
\hline 5 & Belief in information \\
\hline 6 & Perceived reputation \\
\hline
\end{tabular}

\footnotetext{
${ }^{4}$ https://www.ic3.gov/media/2019/190910.aspx
}

\subsection{Context Factors}

We define context as the domain of research. Factors that emerge specifically from the domain of interest and research question of a study can be termed as context factors. In this section, we first briefly discuss three sample contexts along with potential context factors where the PIE framework can be applied. Finally, in Section 4, we select a context with high social impact fake news - and walk through the use of the context factors in the experimental design.

\subsubsection{Information Security}

Cyber-attacks have been a serious challenge for researchers as well as in the industry for several years now. In 2019, the Federal Bureau of Investigation (FBI) reported that email related scams alone exceeded \$26 Billion ${ }^{4}$. Hacker forums and darknet marketplaces play an important role in how malicious attacks are perpetrated [40]. Hackers form semi-structured online networks in order to exchange information and help one another [41]. Hacker posts have been analyzed using sentiment analyzers to better understand their content. Beyond financial motives, hackers are also motivated by political activism [42]. Furthermore, to increase the influence, hackers rely on reputation. Online reputation of hackers is based on several factors, such as size of network, age on forum, and number of messages [43]. Although several empirical techniques have been deployed to understand hacker forum content and predict hacker behavior, little attention has been paid to the role of emojis. Although emoji usage on the dark web is very popular as shown in Figure 3, it still remains an understudied phenomenon. There are multiple attack effects: Confidentiality, Integrity, and Availability (CIA) [44] which affect the information system differently. While confidentiality and integrity violations are caused by an unauthorized user accessing data and affect the content of the data, availability restricts authorized users from accessing data [44]. There are also multiple platforms that could be attacked, including Windows, MAC, and Linux as platform specific malware are very common on the dark web [45]. Finally, multiple products could be being moved in the darkweb including malware and tutorials [46]. Malware is the tool used for carrying out a cyber-attack [46], while tutorial is the write up to do carding, or launch an attack on a specific platform [47]. The PIE framework can be utilized to detect malicious posts across platforms on the dark web using these context factors reported in Table 4 and PIE factors (specifically valence) in Table 1. 


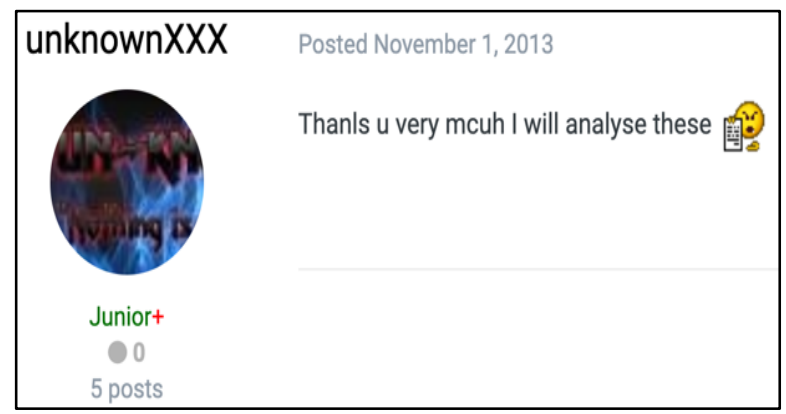

Figure 3. Usage of emoji by an anonymous hacker on a hacker forum discussing malware analysis. The commitment of the junior hacker can be assessed by seriousness seen in the emoji (although, in 2013, the date of this message, emojis were not a part of Unicode consortium yet)

\begin{tabular}{|l|l|l|}
\hline \multicolumn{1}{|c|}{$\begin{array}{c}\text { Context } \\
\text { Factors }\end{array}$} & \# of Levels & \multicolumn{1}{c|}{$\begin{array}{c}\text { Level } \\
\text { Description }\end{array}$} \\
\hline Attack Effect & 3 & $\begin{array}{l}\text { Confidentiality } \\
\text { Integrity } \\
\text { Availability }\end{array}$ \\
\hline $\begin{array}{l}\text { Platform } \\
\text { Attacked }\end{array}$ & 3 or more & $\begin{array}{l}\text { Windows } \\
\text { MAC } \\
\text { Linux }\end{array}$ \\
\hline Product & 2 or more & $\begin{array}{l}\text { Malware } \\
\text { Tutorials }\end{array}$ \\
\hline
\end{tabular}

Table 4. Potential contextual factors in the information security domain

\subsubsection{E-commerce}

Emotions play a strong role in various fields such as marketing [48], mediated communication [49], and advertising, [50] through various mediums (e.g., source, product), and have an effect on beliefs and intentions $[48,51]$. In this context, we can utilize the PIE factor replacement, to measure if words from product descriptions can be substituted to add more emotion for readers to understand the product review or product description better. Some potential context factors include product type (hedonistic/utilitarian) [7], product category [52], and brand personality (humanization of the brand using mascots) [52]. These context factors, when combined with PIE factors such as emoji modifiers that help in self-representation, can help us in understanding how brand perceptions and product consumptions change when emojis are used. Potential context factors for e-commerce are in Table 5. Several other contextual factors in the ecommerce domain can be explored in future analyses.

\begin{tabular}{|l|l|l|}
\hline \multicolumn{1}{|c|}{$\begin{array}{c}\text { Context } \\
\text { Factors }\end{array}$} & \# of Levels & \multicolumn{1}{c|}{$\begin{array}{c}\text { Level } \\
\text { Description }\end{array}$} \\
\hline Product Type & 2 & $\begin{array}{l}\text { Hedonistic } \\
\text { Utilitarian }\end{array}$ \\
\hline $\begin{array}{l}\text { Product } \\
\text { Category }\end{array}$ & 2 or more & $\begin{array}{l}\text { Books } \\
\text { Electronics, } \\
\text { Clothing }\end{array}$ \\
\hline $\begin{array}{l}\text { Brand } \\
\text { Personality }\end{array}$ & 2 & $\begin{array}{l}\text { Humanized } \\
\text { Not Humanized }\end{array}$ \\
\hline
\end{tabular}

Table 5. Potential contextual factors in ecommerce domain A combination of one or more product type along with different product categories can be used with multiple PIE factors

\subsubsection{Social Media Content}

Social media platforms, such as YouTube, contain a significant amount of user generated content. Individuals and groups use these platforms to monetize their content [53]. Crowdsourced platforms (patreon.com), Super Chats and Super Stickers are popular techniques used by content creators on YouTube [53]. Introduced in 2017, Super Stickers are animated pictographs that viewers of live stream can purchase and send them on a live chat ${ }^{5}$ as shown in Figure 4 . These are typically utilized to highlight the viewer's message or express their emotions. Such features indicate the financial value of emojis and pictographs on these platforms. The emoji modifier PIE factor can play a strong role here as well and help increase the financial value of the ideogram. As mentioned earlier, since emoji modifiers play a strong role in self-determination and bring a sense of community, super stickers can improve how viewers express their trust and feelings for content creators on YouTube. The financial impact of different emojis can be studied using context factors in Table 6 (note: this is not an exhaustive list of potential context factors of interest). Payments are driven by several factors reviewed by the social media platform ${ }^{6}$ and depend on several factors such as the main theme, country, and number of hours spent by viewers on the channel [54].

\footnotetext{
${ }^{5}$ https://support.google.com/youtube/answer/9277801

${ }^{6}$ https://support.google.com/youtube/answer/1311392?hl=en
} 


\begin{tabular}{|l|l|l|}
\hline $\begin{array}{c}\text { Context } \\
\text { Factors }\end{array}$ & \multicolumn{1}{|c|}{$\begin{array}{c}\text { \# of } \\
\text { Levels }\end{array}$} & \multicolumn{1}{|c|}{ Level Description } \\
\hline $\begin{array}{l}\text { Main } \\
\text { Theme }\end{array}$ & 2 or more & $\begin{array}{l}\text { Gaming } \\
\text { Fitness } \\
\text { Vines }\end{array}$ \\
\hline $\begin{array}{l}\text { Channel } \\
\text { Subscriber } \\
\text { Level }\end{array}$ & 2 or more & $\begin{array}{l}\text { Silver (at least 100,000 } \\
\text { subscribers) } \\
\text { Gold (at least 1 million } \\
\text { subscribers) } \\
\text { Diamond (at least 10 } \\
\text { million subscribers) }\end{array}$ \\
\hline $\begin{array}{l}\text { Country or } \\
\text { Region }\end{array}$ & 2 or more & $\begin{array}{l}\text { Countries and regions } \\
\text { where this feature is } \\
\text { available. }\end{array}$ \\
\hline
\end{tabular}

Table 6. Potential contextual factors in Social Media Content domain

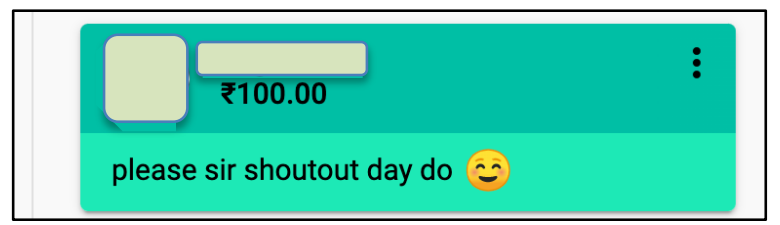

(a)

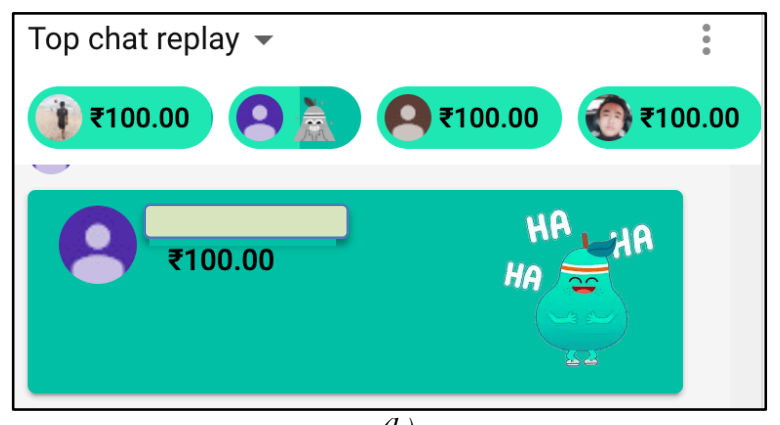

(b)

Figure 4 (a) Super chat with emoji used on a YouTube live chat, (b) Super Sticker being used on a YouTube live chat.

The numbers indicate the amount of money paid in local currency of the live stream location to publish the super chat and super sticker.

\section{Fake News}

Fake News has emerged as a serious challenge for the digital world [55]. Fake news researchers are concerned with measuring the spread of misinformation $[56,57]$. In this section we discuss the impact of emoji usage to understand fake news propagation better. In particular, we describe a mixed design experiment to determine if an ideogrammatic icon affects the likelihood of the communication text going viral, and whether it differs for true news versus fake news.

The experiment design and control variables are based on PIE factors discussed in Table 1, context factors are based on Table 7, and dependent variables are based on Table 3. Figure 5 represents the PIE factor stack combined with fake news factors stack. Here, emoji replacement is a between-subject factor and emoji valence, sources, and veracity of information are withinsubject factors. We first create three conditions for this experiment as shown in Figure 6. The first is a control condition where a tweet has only text and no emojis; in the second condition, we use emojis to describe one or more keywords in the message; the third condition is where the emoji(s) are augmented using the help of keywords. Based on these conditions we describe propositions for the dependent variables discussed in Table 3.

\begin{tabular}{|l|l|l|}
\hline \multicolumn{1}{|c|}{$\begin{array}{c}\text { Context } \\
\text { Factors }\end{array}$} & \multicolumn{1}{|c|}{$\begin{array}{c}\text { \# of } \\
\text { Levels }\end{array}$} & \multicolumn{1}{|c|}{ Level Description } \\
\hline Sources & 2 & $\begin{array}{l}\text { Online news sources } \\
\text { that have been rated } \\
\text { either pro-left or } \\
\text { pro-right }\end{array}$ \\
\hline $\begin{array}{l}\text { Veracity of } \\
\text { Information }\end{array}$ & 2 & $\begin{array}{l}\text { True news is factually } \\
\text { correct information, } \\
\text { Fake news is factually } \\
\text { incorrect information }\end{array}$ \\
\hline $\begin{array}{l}\text { Perceived } \\
\text { Qrgument }\end{array}$ & $\begin{array}{l}\text { 7-point } \\
\text { likert } \\
\text { scale or } 3 \\
\text { levels }\end{array}$ & $\begin{array}{l}\text { Low quality arguments } \\
\text { with no substantive } \\
\text { arguments, medium } \\
\text { quality arguments that } \\
\text { contain some strong } \\
\text { points but not enough to } \\
\text { convince readers, high } \\
\text { quality arguments } \\
\text { backed by facts and } \\
\text { reliable sources }\end{array}$ \\
\hline
\end{tabular}

Table 7. Potential contextual factors for fake news domain

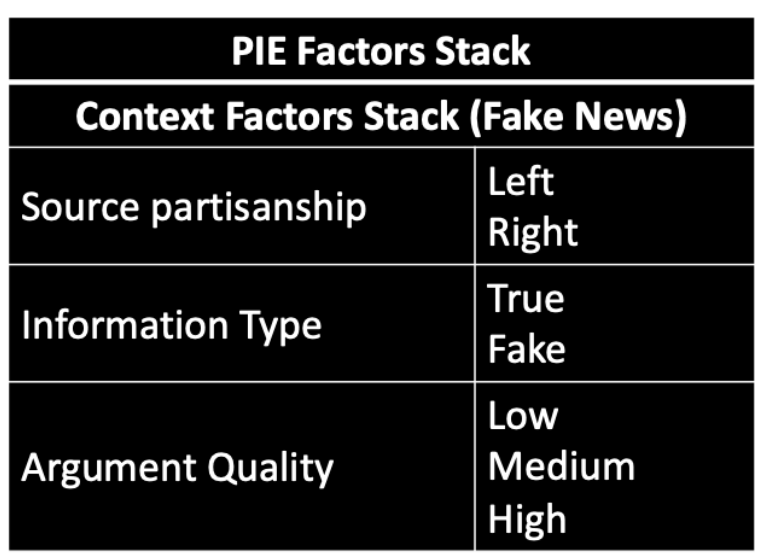

Figure 5. PIE Factors stack placed on top of contextual factor stack for fake news context 


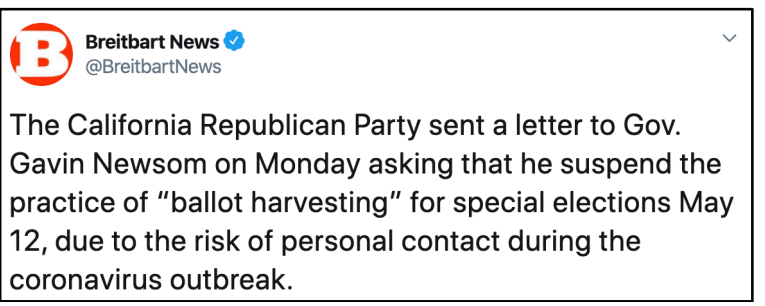

(a)

Breitbart News
The California Republican Party sent a letter to Gov.
Gavin Newsom on Monday asking that he suspend the
practice of "ballot harvesting" for special elections May
12, due to the risk of personal contact during the
coronavirus $-€$ outbreak.

(b)

Breitbart News
The California Republican Party sent a letter to Gov.
Gavin Newsom on Monday asking that he suspend the
practice of "ballot harvesting" for special elections May
12 , due to the risk of personal contact during the 6
outbreak.

(c)

Figure 6. a) Only word (control condition), b) Emoji does not replace words, c) Emoji replaces the word (coronavirus)

Proposition 1: When Emoji replaces a word in a message, it will affect believability differently for different types of emojis. This relationship will be moderated by the veracity of the news.

Proposition 2: When Emoji replaces a word in a message, it will affect the likelihood of sharing differently for different types of emojis. This relationship will be moderated by the veracity of the news.

Proposition 3: When Emoji replaces a word in a message, it will affect the degree of emotional arousal differently for different types of emojis. This relationship will be moderated by the veracity of the news.

\section{Conclusion}

Emojis, also known as pictographs, or ideograms, or paralanguage are used widely on the Internet, both in computer mediated communication and social networking sites. They are used to modify decision making by manipulating emotions similar to facial expressions in face to face communication. There are certain universal properties of emojis (e.g., valence, emotion conveyed) that apply across several contexts and help understand the role of emotions in these contexts. In this study, we discussed three contexts (information security, ecommerce, and social media content) briefly and fake news context in detail. Using examples, we demonstrated how important research questions in these domains can be answered using foundational emoji factors (PIE factors) along with contextual factors. In the fake news context, we provided examples of how replacement of words with emojis can be used to measure intent due to the difference in cognitive load. In the social media content context, we showed how monetization of emojis can be understood and improved. Additionally, we also showed the usage of emojis and their valence to build trust, measured reputation of hackers, or detect malicious posts on the dark web. Finally, we shared a detailed experimental procedure showing how the PIE framework can be used to in the context of fake news. This study serves as a framework for future studies that intend to understand the impact of emojis in the context of their domains, as it can be applied across several other contexts (e.g., cyberbullying) with ease.

\section{References}

[1] S. W. Littlejohn and K. A. Foss, Theories of Human Communication. 2010.

[2] N. Tahmasbi and E. Rastegari, A Socio-contextual Approach in Automated Detection of Cyberbullying.

[3] M. Janson, J. Iivari, and H. Oinas-Kukkonen, "Association for Information Systems AIS Electronic Library (AISeL)Mediated Social Action Recommended Citation eCommerce as ComputerMediated Social Action," 2000.

[4] M. Zuckerman, B. M. Depaulo, and R. Rosenthal, "Verbal and nonverbal communication of deception," Advances in Experimental Social Psychology, vol. 14, no. C, pp. 1-59, Jan. 1981, doi: 10.1016/S0065-2601(08)60369-X.

[5] M. Danesi, The semiotics of emoji: The rise of visual language in the age of the internet. 2016.

[6] M. Davis and P. Edberg, "Unicode \& Emoji," 2015.

[7] G. Das, H. Wiener, and I. Kareklas, "To emoji or not to emoji? Examining the influence of emoji on consumer reactions to advertising," Journal of Business Research, 2019.

[8] J. Whitney, M. Jennex, A. Elkins, and E. Frost, "Don't Want to Get Caught? Don't Say It: The Use of EMOJIS in Online Human Sex Trafficking Ads," in Proceedings of the 51st Hawaii International Conference on System Sciences, 2018, doi: 10.24251/hicss.2018.537.

[9] F. Barbieri, M. Ballesteros, and H. Saggion, Are emojis predictable?, vol. 2. Association for Computational Linguistics (ACL), 2017.

[10] J. B. Walther and K. P. D'addario, "The Impacts of Emoticons on Message Interpretation in Computer- 
Mediated Communication," Social science computer review, vol. 19, no. 3, pp. 324-347, 2001.

[11] P. K. Novak, J. Smailović, B. Sluban, and I. Mozetič, "Sentiment of Emojis," 2015, doi: 10.1371/journal.pone.0144296.

[12] H. J. Wall, L. K. Kaye, and S. A. Malone, “An exploration of psychological factors on emoticon usage and implications for judgement accuracy," 2016, doi: 10.1016/j.chb.2016.03.040.

[13] L. K. Kaye, S. A. Malone, and H. J. Wall, "Emojis: Insights, Affordances, and Possibilities for Psychological Science," Trends in cognitive sciences, 21(2), 66-68., vol. 21, no. 2, pp. 66-68, 2017, doi: 10.1016/j.tics.2016.10.007.

[14] J. B. Walther, "Selective self-presentation in computer-mediated communication: Hyperpersonal dimensions of technology, language, and cognition," Computers in Human Behavior, vol. 23, pp. 25382557, 2007, doi: 10.1016/j.chb.2006.05.002.

[15] S. Kiesler, J. Siegel, and T. W. Mcguire, "Social Psychological Aspects of Computer-Mediated Communication," 1984.

[16] M. Lea and R. Spears, "Paralanguage and social perception in computer-mediated communication," Journal of Organizational Computing and Electronic Commerce, vol. 2, pp. 321-341, 1992, doi: 10.1080/10919399209540190.

[17] M. Spitzer, "WRITING STYLE IN COMPUTER CONFERENCES.," IEEE Transactions on Professional Communications, vol. PC-29, no. 1, pp. 19-22, Mar. 1986, doi: 10.1109/TPC.1986.6449010.

[18] R. T. Craig, "Communication Theory as a Field," Communication Theory, vol. 9, no. 2, pp. 119-161, May 1999, doi: 10.1111/j.1468-

2885.1999.tb00355.x.

[19] C. Morris, "Foundations of the Theory of Signs," pure.mpg.de, 1938.

[20] H. Miller, J. Thebault-Spieker, S. Chang, I. Johnson, L. Terveen, and B. Hecht, “'Blissfully Happy' or 'Ready toFight': Varying Interpretations of Emoji,' 2016.

[21] S. Annamalai, S. Nur, and A. Salam, "Undergraduates' Interpretation on WhatsApp Smiley Emoji," Jurnal Komunikasi Malaysian Journal of Communication Jilid, vol. 33, no. 4, p. 2017, Dec. 2017, doi: 10.17576/JKMJC-2017-330406.

[22] K. Tracy and K. Haspel, "Language and Social Interaction: Its Institutional Identity, Intellectual Landscape, and Discipline-Shifting Agenda," Journal of Communication, vol. 54, no. 4, pp. $788-$ 816, Dec. 2004, doi: 10.1111/j.14602466.2004.tb02654.x.

[23] P. Ekman, "Are There Basic Emotions?," Psychological Review, vol. 99, no. 3, pp. 550-553, 1992, doi: 10.1037/0033-295X.99.3.550.

[24] N. Na'aman, H. Provenza, and O. Montoya, "MojiSem: Varying linguistic purposes of emoji in (Twitter) context," in Proceedings of ACL 2017, Student Research Workshop, 2017, pp. 136-141, doi: 10.18653/v1/P17-3022.
[25] J. B. Walther, J. F. Anderson, and D. W. Park, "Interpersonal Effects in Computer-Mediated Interaction: A Meta-Analysis of Social and Antisocial Communication," Communication Research, vol. 21, no. 4, pp. 460-487, 1994, doi: 10.1177/009365094021004002.

[26] D. Derks, A. E. R. Bos, and J. Von Grumbkow, "Emoticons in Computer-Mediated Communication: Social Motives and Social Context," CYBERPSYCHOLOGY \& BEHAVIOR, vol. 11, no. 1, 2008, doi: 10.1089/cpb.2007.9926.

[27] J. Kruger, N. Epley, J. Parker, and Z. W. Ng, "Egocentrism over E-mail: Can we communicate as well as we think?," Journal of Personality and Social Psychology, vol. 89, no. 6, pp. 925-936, Dec. 2005, doi: 10.1037/0022-3514.89.6.925.

[28] I. D. Wood and S. Ruder, "Emoji as Emotion Tags for Tweets," in Proceedings of the Emotion and Sentiment Analysis Workshop LREC, 2016.

[29] X. Li, K. W. Chan, and S. Kim, "Service with Emoticons: How Customers Interpret Employee Use of Emoticons in Online Service Encounters," Journal of Consumer Research, vol. 45, no. 5, pp. 973-987, 2019, doi: 10.1093/jcr/ucy016.

[30] J. B. Walther, "Interpersonal Effects in ComputerMediated Interaction: A Relational Perspective," Communication Research, vol. 19, no. 1, pp. 52-90, 1992, doi: 10.1177/009365092019001003.

[31] S. K. Lo, "The nonverbal communication functions of emoticons in computer-mediated communication," Cyberpsychology and Behavior, vol. 11, no. 5, pp. 595-597, Oct. 2008, doi: 10.1089/cpb.2007.0132.

[32] N. Cohn, "A multimodal parallel architecture: A cognitive framework for multimodal interactions," Cognition, vol. 146, pp. 304-323, Jan. 2016, doi: 10.1016/j.cognition.2015.10.007.

[33] D. McNeill, Language and Gesture. Cambridge University Press., 2000.

[34] V. Gustafsson, "Replacing words with emojis and its effect on reading time," USCCS, p. 73, 2017.

[35] F. Barbieri, M. Ballesteros, F. Ronzano, and H. Saggion, "Multimodal Emoji Prediction," 2018, pp. 679-686, doi: 10.18653/v1/n18-2107.

[36] R. R. Provine, R. J. Spencer, and D. L. Mandell, "Emotional Expression Online Emoticons Punctuate Website Text Messages," Journal of Language and Social Psychology, vol. 26, no. 3, 2007, doi: 10.1177/0261927X06303481.

[37] T. Fitzpatrick, "The validity and practicality of sunreactive skin types I through VI," Archives of Dermatology, vol. 124, no. 6, pp. 869-871, 1988.

[38] A. Robertson, W. Magdy, and S. Goldwater, "SelfRepresentation on Twitter Using Emoji Skin Color Modifiers," 2018.

[39] F. Barbieri and J. Camacho-Collados, "How Gender and Skin Tone Modifiers Affect Emoji Semantics in Twitter," in Proceedings of the 7th Joint Conference on Lexical and Computational Semantics, 2018, pp. 101-106.

[40] E. Nunes, A. Diab, A. Gunn, E. Marin, and P. 
Shakarian, "Darknet and deepnet mining for proactive cybersecurity threat intelligence," in IEEE Conference on Intelligence and Security Informatics (ISI), 2016, pp. 7-12.

[41] T. J. Holt, "Deviant Behavior subcultural evolution? examining the influence of on-and off-line experiences on deviant subcultures," 2007, doi: 10.1080/01639620601131065.

[42] M. Macdonald, R. Frank, J. Mei, and B. Monk, "Identifying Digital Threats in a Hacker Web Forum," dl.acm.org, pp. 926-933, Aug. 2015, doi: 10.1145/2808797.2808878.

[43] D. Décary-Hétu and B. Dupont, "Global Crime Reputation in a dark network of online criminals," 2013, doi: 10.1080/17440572.2013.801015.

[44] S. Samonas and D. Coss, "THE CIA STRIKES BACK: REDEFINING CONFIDENTIALITY, INTEGRITY AND AVAILABILITY IN SECURITY," Journal of Information System Security, vol. 10, no. 3, 2014.

[45] M. H. Ligh, A. Case, J. Levy, and Aa. Walters, "The Art of Memory Forensics: Detecting Malware and Threats in Windows, Linux," John Wiley \& Sons, 2014. [Online]. Available: https://books.google.com/books?hl=en\&lr=\&id=U1j OAwAAQBAJ\&oi=fnd\&pg=PR17\&dq=malware+ \%22linux\%22\&ots=yixFYAYT2i\&sig=Lo117Q1P7 S-pV0xVsNQLotb2bSY\#v=onepage \& $\mathrm{q}=$ malware $\% 22$ linux\%22\&f=false. [Accessed: 15-Jul-2020].

[46] P.-Y. Du et al., "Identifying, collecting, and presenting hacker community data: Forums, IRC, carding shops, and DNMs," in IEEE international conference on intelligence and security informatics (ISI), 2018, pp. 70-75.

[47] S. Samtani, R. Chinn, H. Chen, and J. F. Nunamaker Jr, "Exploring Emerging Hacker Assets and Key Hackers for Proactive Cyber Threat Intelligence," Journal of Management Information Systems, vol. 34, no. 4, pp. 1023-1053, Oct. 2017, doi: $10.1080 / 07421222.2017 .1394049$.
[48] R. P. Bagozzi, M. Gopinath, and P. U. Nyer, "The Role of Emotions in Marketing," 1999.

[49] S. Brown, R. Fuller, and S. Thatcher, "Impression formation and durability in mediated communication," Journal of the Association for Information Systems, vol. 17, no. 9, p. 1, 2017.

[50] M. B. Holbrook and J. O'Shaughnessy, "The role of emotion in advertising," Psychology and Marketing, vol. 1, no. 2, pp. 45-64, 1984, doi: 10.1002/mar.4220010206.

[51] S. P. Brown, W. L. Cron, and J. W. Slocum, "Effects of Goal-Directed Emotions on Salesperson Volitions, Behavior, and Performance: A

Longitudinal Study," Journal of Marketing, vol. 61, no. 1, pp. 39-50, Jan. 1997, doi: $10.1177 / 002224299706100104$

[52] A. W. Luangrath, J. Peck, and V. A. Barger, "Textual paralanguage and its implications for marketing communications is," 2016, doi: 10.1016/j.jcps.2016.05.002.

[53] K. Munger and J. Phillips, "A supply and demand framework for YouTube politics," Preprint, 2019.

[54] C. Welch, "YouTube tightens rules around what channels can be monetized," The Verge, 2018. [Online]. Available: https://www.theverge.com/2018/1/16/16899068/you tube-new-monetization-rules-announced-4000hours. [Accessed: 15-Jul-2020].

[55] S. Vosoughi, D. Roy, and S. Aral, "The spread of true and false news online," science, vol. 359, no. 6380, pp. 1146-1151, 2018.

[56] S. Suntwal, S. A. Brown, and M. W. Patton, "How does Information Spread? A Study of True and Fake News," in Proceedings of the 53rd Hawaii International Conference on System Sciences., 2020.

[57] A. Kim and A. R. Dennis, "Says who? The effects of presentation format and source rating on fake news in social media.," MIS Quarterly, vol. 43, no. 3, 2019. 